(2) Open Access Full Text Article

REVIEW

\title{
Assessment and monitoring of biologic drug adverse events in patients with psoriasis
}

\author{
This article was published in the following Dove Press journal: \\ Psoriasis: Targets and Therapy \\ I April 2016 \\ Number of times this article has been viewed
}

\section{Tessa Hanley \\ Marc Handford \\ Dawn Lavery \\ Zenas ZNYiu}

Dermatology Centre, Salford Royal NHS Foundation Trust, Manchester, UK
Background: Current treatment guidelines for biologic therapies in psoriasis differ in their recommendation for the monitoring of adverse events.

Objective: The aim of this paper was to draw together evidence from the currently available guidelines as a summary of how biologics licensed for the treatment of psoriasis should be monitored for adverse events.

Methods: The MEDLINE database was searched to identity the current literature on the safety and screening guidance associated with infliximab, etanercept, adalimumab, ustekinumab, and secukinumab.

Limitations: This study was limited by the lack of data evaluating monitoring in patients with psoriasis undergoing treatment with a biologic therapy.

Results: This review of the current literature highlights that there are areas of routine screening, which are recommended in current practice, which require further evidence to investigate its true utility.

Conclusion: Most screening and monitoring tests performed routinely in clinical practice are supported by minimal clinical evidence, highlighting the need for more studies to evaluate the role and value of the different modalities of screening and monitoring for adverse events in those with psoriasis receiving treatment with biologic therapies.

Keywords: biologics, monitoring, psoriasis, screening, safety

\section{Background}

Psoriasis is a common, chronic, inflammatory skin disease affecting between $1 \%$ and $3 \%$ of the world population. ${ }^{1}$ It is characterized by erythematous pruritic plaques and associated with a significant impairment in the quality of life akin to other chronic medical conditions, eg, diabetes. ${ }^{2}$ Psoriasis is also associated with a number of other chronic conditions, including cardiovascular disease, depression, and metabolic syndrome, ${ }^{1,3}$ while $\sim 25 \%$ of patients also suffer from psoriatic arthritis. ${ }^{4}$ Management of psoriasis should therefore be holistic, timely, and effective and provide long-term disease control. ${ }^{5}$

Traditional standard systemic therapies for the treatment of moderate-to-severe psoriasis include methotrexate, ciclosporin, acitretin, and fumaric acid esters. These treatments can be ineffective and are associated with long-term adverse effects. ${ }^{5}$

The biologic therapies offer a potent treatment alternative to patients with moderate-to-severe psoriasis. They block specific cytokine pathways pertinent to the pathogenesis of psoriasis, and there is a substantial body of evidence to support the use of these agents. ${ }^{5}$ The National Institute for Health and Care Excellence (NICE),
Dermatology Centre, Salford Royal NHS Foundation Trust, Stott Lane, Manchester M6 8HD, UK

Tel +44 I6I 3060620

Email zenas.yiu@manchester.ac.uk
Psoriasis: Targets and Therapy 20।6:6 4I-54

(c) (1) (8) ( 2016 Hanley et al. This work is published and licensed by Dove Medical Press Limited. The full terms of this license are avallable at https://www.dovepress.com/terms. cc) you herbby accept the Terms. Non-commercial uses of the work are permitted without any further permision from Dove Medical Press Limited, provided the work is properly attributed. For

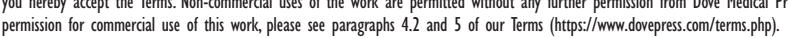

Dovepress

http://dx.doi.org/10.2147/PTT.S68869

41 
a national guidance body in the UK, has now recommended five biologic therapies for the treatment of moderate-to-severe psoriasis, which include etanercept, infliximab, adalimumab, and ustekinumab, with secukinumab that was recommended for severe plaque psoriasis as of July 2015 . $^{3}$

Although the efficacy of the biologic therapies has been well established through clinical trial data, there is a significant uncertainty regarding the incidence and prevalence of short- and long-term adverse effects of these drugs and how they should be monitored. This is due to the fact that most of the published clinical trials have insufficient sample sizes to detect rare adverse events and/or were conducted for a short period of time. Trial extensions were often conducted without a control arm, making it difficult to assess the incidence of adverse events of these treatments in the moderate-to-severe psoriasis population. In addition, most trials excluded patients with significant comorbidities resulting in a poor external validity for the safety profile of these drugs in the real-world clinic. ${ }^{6}$ The potential for any drug to produce rare but significant adverse effects was demonstrated by the experience with efalizumab, which was withdrawn from the market in 2009 by the European Medicines Agency following its association with progressive multifocal leukoencephalopathy through the analysis of postmarketing pharmacovigilance data. ${ }^{7,8}$ The safety profile of biologic therapies, therefore, would be best evaluated through the interrogation of observational data, such as prospectively established treatment registries. In the UK, NICE recommends that all patients starting treatment with biologics are invited to participate in the British Association of Dermatologists Biologic Interventions Register (BADBIR). ${ }^{9}$ The BADBIR is a prospective pharmacovigilance study setup to assess the long-term safety of biologic treatments for psoriasis, comparing patients on biologic therapies with a similar patient control group on conventional systemic agents. ${ }^{9,10}$

Understanding the risks of adverse events of biologic therapies is crucial for consent, safe prescribing, and monitoring of these drugs.

\section{Objective}

The aim of this paper was to review the current literature surrounding the potential adverse effects of the five biologics commonly used in the treatment of moderate-to-severe psoriasis and draw together evidence from international guidelines as a summary of how these drugs should be monitored.

\section{Methods}

The MEDLINE database was searched for articles pertaining to biologic agents in the treatment of psoriasis/psoriatic arthritis. Key search terms included: psoriasis/psoriatic arthritis, safety, screening, and biologics (search included trade names). English language articles published between 2000 and 2015 were reviewed, whereas single case reports were discarded. Articles identified within citations from reviewed articles were also reviewed for inclusion.

\section{Introduction}

The current guidance on screening and monitoring from the British Association of Dermatologists (BAD), the European Academy of Dermatology and Venereology (EADV), and the American Academy of Dermatology (AAD) is highlighted together in Table 1. The guidance on each separate category is reviewed in detail with a following discussion, and the conclusions of which are drawn together at the end of the paper.

\section{Overview of serious adverse effects of biologics Serious infection}

Tumor necrosis factor (TNF)- $\alpha$ is needed for formation and maintenance of granulomas; its inhibition can therefore increase the risk of infections such as tuberculosis (TB). TNF- $\alpha$ is also involved in macrophage activation, macrophage differentiation, and phagosome formation, therefore, holding a critical role in the clearance of intracellular pathogens. Furthermore, TNF inhibitors can cause neutropenia and increasing risk of bacterial infection and reduce the immune response to viral pathogens. ${ }^{11}$

Data surrounding serious infection in patients with psoriasis are limited. The psoriasis longitudinal assessment and registry, which is a large, pharmacovigilance registry based in the USA and Europe for patients with psoriasis, run by the drug company Janssen Cilag contains data from 12,095 patients. ${ }^{12}$ When looking at TNF inhibitors and ustekinumab combined, the risk of developing a serious infection was determined to be 1.45 per 100 patient-years, compared with rates of 1.05 and 1.28 per 100 patient-years in nonmethotrexate/nonbiologics and methotrexate/nonbiologic cohorts, respectively. Adalimumab and infliximab therapy was associated with significantly higher incidence of serious infections than other biologics (1.97 and 2.49 per 100 patient-years, respectively), while ustekinumab had the most favorable results with rates of 0.83 serious infections per 100 patient-years. The analysis of data, after adjustment for probable confounders, found that exposure to infliximab or adalimumab was independent risk factors for serious infection as compared with systemic retinoid therapy and/ 
Table I Current guidelines on screening and monitoring of adverse events in patients taking biologics

\begin{tabular}{lll}
\hline $\begin{array}{l}\text { British Association of Dermatologists } \\
\text { Guidelines }-2009^{5}\end{array}$ & $\begin{array}{l}\text { American Academy of } \\
\text { Dermatology Guidelines }-2008^{1}\end{array}$ & $\begin{array}{l}\text { European Academy of Dermatology } \\
\text { and Venereology Guidelines }-2015^{21}\end{array}$ \\
\hline TNF inhibitors & TNF inhibitors & TNF inhibitors/ustekinumab** \\
\hline
\end{tabular}

\section{General infection}

TNF inhibitors contraindicated in patients with active, serious infection

Pretreatment: All patients on biologics should be warned about risk factors for Salmonella and Listeria During treatment: Patients should be monitored for early signs and symptoms of infection throughout the treatment. 3-6 monthly intervals are advised

\section{TB infection}

Active TB is a contraindication to therapy

Pretreatment: All patients should be assessed for active or latent TB before starting biologic therapy CXR and mantoux test (if no immunosuppression in the last 3 months)

\section{CXR and TB ELISpot/QuantiFERON if}

immunosuppressed. Refer all patients with a history of previously treated TB

Those with latent TB should receive treatment prior to initiating therapy

During treatment: Consider risk factors for tuberculosis before treatment and at 3-6 monthly intervals

Annual IGRA if following assessment patient felt to have been exposed to TB

\section{Hepatitis (B and C)}

Pretreatment: Insufficient evidence to justify use of TNF inhibitors in patients with chronic, potentially harmful viral infections (HIV/HBV/HCV/herpes) needs a case-by-case assessment

During treatment: In those with $\mathrm{HCV}$, periodic assessment of viral load

Hepatitis B - periodic assessment for those at risk

\section{Cardiovascular disease}

Pretreatment: Therapy contraindicated in NYHA class III/IV

Echo if well compensated NYHA class I/II - if LVEF

$<50 \%$ consider avoiding biologic therapy
TNF inhibitors contraindicated in patients with active, serious infection

Pretreatment: No specific guidance issued

During treatment: "Periodic" history and examination are recommended

Active TB is a contraindication to therapy

Pretreatment: TB testing (tuberculin skin test) should be performed on all patients before treatment Institutional workers/frequent travelers need repeat screening at "appropriate" intervals. CXR is not indicated

During treatment: Yearly TST testing Institutional workers/frequent travelers need repeat screening at “appropriate” intervals

Pretreatment: Screen for HBV in appropriate clinical setting (reactivation of HBV after TNF inhibitors commenced has been reported). Consultation with liver specialist advised when considering biologics in patients with concomitant $\mathrm{HCV}$

During treatment: No specific guidance issued

Pretreatment: Therapy contraindicated in NYHA class III/IV Echo if well compensated NYHA class I/II - if LVEF $<50 \%$ consider avoiding biologic therapy
Recurrent or severe infections are a relative contraindication for use of all TNF inhibitors

Pretreatment: History and examination for evidence of infection

During treatment: Clinical assessment for risk factors of serious infection - frequency of assessment is not stated

**Contraindicated in active infections

Active TB is a contraindication to all TNF inhibitor therapy

Pretreatment: Prescreening: guidelines recommend anamnesis, a CXR, tuberculin skin test, and QuantiFERON

During treatment: Recommend annual rescreening of latent TB (even if latent TB has previously been correctly treated) using clinical history, TST, and IGRA testing

Pretreatment: With regard to prior/current hepatitis $B$ infection and current chronic hepatitis $C$ infection - treatment guidelines advise consultation with gastroenterologist or hepatologist before initiating the treatment

Guidelines also give drug-specific guidance as follows:

I. Adalimumab and infliximab active chronic $\mathrm{HBV}$ is an absolute contraindication to use; $\mathrm{HCV}$ is a relative contraindication to use

2. Etanercept - chronic active HBV and $\mathrm{HCV}$ are relative contraindications to use and antiretroviral drugs are recommended if biologic therapy is to be initiated

During treatment: No specific guidance issued. **Treatment guidelines advise consultation with gastroenterologist or hepatologist before initiating the treatment

Pretreatment: History and examination for evidence of congestive heart failure NYHA class III/IV is a contraindication for all TNF inhibitors 
Table I (Continued)

\begin{tabular}{l} 
British Association of Dermatologists \\
Guidelines - 20095 \\
\hline TNF inhibitors \\
\hline During treatment: Monitoring at 3-6 months \\
Neurological disease \\
Pretreatment: Avoid in patients with a history of \\
demyelinating disease \\
Use in caution in patients with first-degree relative \\
with demyelinating disease
\end{tabular}
with demyelinating disease

During treatment: Withdraw drug if symptoms are suggestive of demyelination Monitoring at 3-6 months

\section{Malignancy}

Biologic therapy is relatively contraindicated in patients with a history of prior therapy with PUVA $(>200)$ and/or UVB (>350)

Pretreatment: Ensure concordant with national screening programs

Take history of past or present malignancy - TNF inhibitors are relatively contraindicated in patients with a malignancy in the past 5 years

During treatment: Monitor every 3 months

\section{Vaccinations}

Pretreatment: Vaccination should be reviewed and brought up prior to initiation of biologic therapy with reference to the Department of Health Guidance

Patients should not receive live or attenuated vaccines $<2$ weeks before of 6 months after discontinuation of therapy

Inactivated vaccines are safe to administer concurrently, but ideally should be given 2 weeks prior to starting therapy for an optimal immune response

During treatment: Patients should be advised to receive the pneumococcal and annual influenza vaccine during treatment

\section{Laboratory testing}

Pretreatment: FBC, U\&E, LFTs, hepatitis B/hepatitis $\mathrm{C} / \mathrm{HIV}$ serology, autoantibodies including ANA and anti-dsDNA

Beta-HcG in females if believed to be at risk of pregnancy

\begin{tabular}{|c|c|}
\hline $\begin{array}{l}\text { American Academy of } \\
\text { Dermatology Guidelines - 2008' }\end{array}$ & $\begin{array}{l}\text { European Academy of Dermatology } \\
\text { and Venereology Guidelines - } 2015^{21}\end{array}$ \\
\hline TNF inhibitors & TNF inhibitors/ustekinumab** \\
\hline $\begin{array}{l}\text { During treatment: "Periodic" } \\
\text { history and examination are } \\
\text { recommended }\end{array}$ & $\begin{array}{l}\text { During treatment: Clinical assessment for } \\
\text { signs of congestive heart failure - frequency } \\
\text { of assessment not stated }\end{array}$ \\
\hline
\end{tabular}

Pretreatment: Contraindicated in patients with MS or other demyelinating disease

Evidence "strongly suggests" avoidance in patients with firstdegree relatives with MS

During treatment: "Periodic" history and examination are recommended

Withhold TNF inhibitors if evidence of demyelinating disease

Pretreatment: Carefully consider use in patients with history of malignancy (particularly lymphoma) Consider potential risk of T-cell lymphoma, melanoma, and nonmelanoma skin cancer

During treatment: "Periodic" history and examination are recommended

Pretreatment: Standard vaccinations including pneumococcal, hepatitis $A$ and $B$, influenza, and tetanus are recommended prior to initiation of therapy

Once the treatment has begun, live vaccines and live attenuated vaccines are to be avoided in all circumstances

During treatment: Physicians should consider the advantages and disadvantages of killed virus vaccines such as influenza

Pretreatment: FBC, LFTs, and hepatitis profile - including hepatitis $B$ and hepatitis $C$ serology
TNF inhibitors are not recommended in patients with MS or other demyelinating disease Use TNF inhibitors with caution in patients with a first-degree relative with a demyelinating disease

Pretreatment: History and examination for evidence of neurological symptoms During treatment: Clinical assessment for neurological symptoms - frequency of assessment not stated

**Can be used in patients with coexisting neurological disease

PUVA ( $>200$ treatments) is a relative contraindication to treatment with all TNF inhibitors

Malignancies and lymphoproliferative disorders are a relative contraindication for all TNF inhibitors - in patients with current cancer or cancer in the past 5 years, treatment decision has to be made on a case-by-case basis following discussion with a cancer specialist

Pretreatment: History and examination During treatment: Clinical assessment focusing on lymphadenopathy, malignancies (especially skin cancer), and premalignant lesions - frequency of assessment not stated

Pretreatment: All recommended vaccination should be given prior to any systemic therapy. In general, common vaccinations with nonlive vaccines are safe during treatment with all drugs

Live vaccines and are mostly contraindicated Vaccination status for hepatitis A and B is important and primary vaccination and/ or boostering should be performed before systemic treatment initiation

During treatment: During therapy with all systemic agents annual influenza vaccination is recommended

Pretreatment: FBC, U\&E, LFTs, CRP, hepatitis $\mathrm{B} /$ hepatitis $\mathrm{C} / \mathrm{HIV}$ serology, urinalysis, urine beta-HcG (females) focusing on evidence of malignancy 
Table I (Continued)

\begin{tabular}{|c|c|c|}
\hline $\begin{array}{l}\text { British Association of Dermatologists } \\
\text { Guidelines }-2009^{5}\end{array}$ & $\begin{array}{l}\text { American Academy of } \\
\text { Dermatology Guidelines - 2008' }\end{array}$ & $\begin{array}{l}\text { European Academy of Dermatology } \\
\text { and Venereology Guidelines - } 2015^{21}\end{array}$ \\
\hline TNF inhibitors & TNF inhibitors & TNF inhibitors/ustekinumab*** \\
\hline $\begin{array}{l}\text { During treatment: Repeat FBC, U\&E, and LFTs at } \\
3 \text { months and } 6 \text { months. } \\
\text { Repeat hepatitis B and HIV serology "periodically" } \\
\text { in those at risk. } \\
\text { Autoantibodies if development of autoimmune } \\
\text { disease }\end{array}$ & $\begin{array}{l}\text { During treatment: "Periodic" FBC } \\
\text { and LFTs }\end{array}$ & $\begin{array}{l}\text { During treatment: Etanercept and } \\
\text { adalimumab = FBC, U\&E, LFTs, and } \\
\text { urinalysis at } 4 \text { weeks, I } 2 \text { weeks, and every } \\
3-6 \text { months thereafter. } \\
\text { Infliximab = repeat FBC, U\&E, LFTs, and } \\
\text { urinalysis at } 4 \text { weeks, I2 weeks, and prior } \\
\text { to each infusion } \\
\text { **Pretreatment: As for TNF inhibitors } \\
\text { During treatment: Repeat FBC, U\&E, LFTs, } \\
\text { and urinalysis every 3-6 months }\end{array}$ \\
\hline
\end{tabular}

Note: ** Guidance relating to ustekinumab.

Abbreviations: HBV, hepatitis B virus; HCV, hepatitis C virus; HIV, human immunodeficiency virus; IGRA, interferon gamma release assay; MS, multiple sclerosis; PUVA, 8-methoxypsoralen-ultraviolet A; TB, tuberculosis; TNF, tumor necrosis factor; TST, tuberculin skin test; UVB, ultraviolet B; NYHA, New York Heart Association; CXR, chest X-ray; FBC, full blood count; U\&E, urea and electrolytes; HcG, human chorionic gonadotropin; ANA, anti-nuclear antibodies.

or phototherapy, with hazard ratios of $2.51(95 \%$ confidence interval (CI): $1.45-4.33 ; P<0.001)$ and $2.13(95 \% \mathrm{CI}$ : $1.33-3.41 ; P=0.002)$, respectively. Additional risk factors were found to be increased age and a previous history of serious infection. ${ }^{12}$

A 2012 study by Reich et al evaluated 4 years of pooled safety clinical trial data of ustekinumab in patients with moderate-to-severe psoriasis found that rates of serious infection for doses of ustekinumab (45 mg and $90 \mathrm{mg}$ ) were consistent with the incidence of serious infection in the general population. Cumulative rates per 100 patient-years were 0.8 and 1.32 for doses of $45 \mathrm{mg}$ and $90 \mathrm{mg}$, respectively, concurring with the psoriasis longitudinal assessment and registry results. ${ }^{13}$

Two Phase III, double-blinded trials assessed the safety of secukinumab over a 52-week period. A total of 738 patients were randomly assigned to the Efficacy of Response and Safety of Two Fixed Secukinumab Regimens in Psoriasis (ERASURE) study and 1,306 patients to the Full-Year Investigative Examination of Secukinumab vs Etanercept Using Two Dosing Regimens to Determine Efficacy in Psoriasis (FIXTURE) study. Patients were assigned to secukinumab $300 \mathrm{mg}, 150 \mathrm{mg}$, or placebo, and patients in the FIXTURE study are also assigned to another group of etanercept $50 \mathrm{mg}$. The ERASURE study found serious infection rates to be 1.0, 0.7 , and 1.5 per 100 patient-years for the $300 \mathrm{mg}, 150 \mathrm{mg}$, and placebo groups, respectively. The FIXTURE study showed serious infection rates to be $1.5,0.6$, and 0.3 and 1.2 per 100 patient-years for the $300 \mathrm{mg}, 150 \mathrm{mg}$, and placebo and etanercept groups, respectively. ${ }^{14}$

\section{Guidelines for assessment and monitoring}

Given the mode of action of the biologic therapies, patients are at an increased theoretical risk of infection. Furthermore, there is literature demonstrating an increased risk of infection with adalimumab and infliximab compared with retinoid therapy and/or phototherapy. All patients receiving treatment with a biologic therapy, therefore, need to be actively and routinely monitored for evidence of infection. Current guidelines offer no consensus on how often this should be.

\section{Tuberculosis}

TNF inhibitor treatment has been associated with an increased risk of TB reactivation. ${ }^{7}$ The majority of the data supporting this come from rheumatology studies and registries; however, differences in underlying primary pathology and comorbidities mean that inferring results to patients with psoriasis is difficult.

Sánchez-Moya et al published an analysis of the Spanish registry for systemic biological and nonbiological treatments in psoriasis. He identified 793 patients receiving biologic therapy for psoriasis. Overall follow-up was 3,720 patientyears. Of the 793 patients, 163 (20.5\%) tested positive for latent TB prior to the initiation of therapy. Of the patients with latent TB infection, there was an incidence of active infection of 145 cases per 100,000 patient-years compared with a control group in which there were no cases of active TB infection (95\% CI: 54-389). ${ }^{15}$ These data support the findings of Sánchez-Moya and Dauden and Ergun et al who showed modest TB reactivation rates of $1.08 \%$ (sample size: 370 ) and $2.38 \%$ (sample size: 42 ), respectively, in patients on anti-TNF agents. Neither study had a control group. ${ }^{16,17}$

Ustekinumab is a fully human monoclonal antibody that targets interleukin (IL)-12 and IL-23. It binds to the p40 subunit disrupting the inflammatory cascade implicated in the pathogenesis of psoriasis. ${ }^{18}$ The IL-12 pathway is important in regulating immunity to mycobacterium 
$\mathrm{TB}$, and it is therefore reassuring to note that data from 3,177 patients with psoriasis across five Phase III trials of ustekinumab (45 mg or $90 \mathrm{mg}$ ) found that in patients with a positive tuberculin skin test (TST) or quantiFERON gamma assay there were no cases of latent TB reactivation in those having taken isoniazid prophylaxis. ${ }^{19}$ The analysis of prospective registries in this regard is critical to establishing the true risk of acquired or reactivated TB in patients on ustekinumab.

Secukinumab is a fully human anti-IL-17A monoclonal antibody. ${ }^{3}$ The analysis of pooled data from Phase III clinical trials has shown no evidence of reactivation of previous or latent TB compared with placebo. ${ }^{20}$

It should be noted that clinical TB infection has been shown to vary between the different biologic agents. This has been demonstrated by the examination of case reports to the US Food and Drug Administration, which found the median time between the initiation of therapy and evidence of clinical infection is 3 months, 4-6 months, and 11.5 months for infliximab $(n=70)$, adalimumab $(n=15)$, and etanercept $(n=25)$, respectively. It should be noted that the majority of these infections are the reactivation of latent TB infection..$^{5,21-23}$

\section{Guidelines for assessment and monitoring}

Table 1 highlights the current guidelines with respect to the assessment of TB prior to biologic therapy and monitoring during therapy. As secukinumab is new to the market, there were no specific guidelines at the time of publication. However, NICE concluded that secukinumab did not appear to be associated with adverse events not already known for biological treatments in general, suggesting that the same precautions should be taken pre-therapy as with other biologic therapies. ${ }^{24}$

There are two commonly used screening tests for exposure to TB - the TST and the interferon gamma release assay (IGRA), marketed as QuantiFERON gold. BAD guidelines, published 6 years ago, suggest using the IGRA only in immunosuppressed patients. However, studies that have compared the two methods of screening have shown the IGRA to have higher sensitivity and specificity. ${ }^{5,25}$ A 2008 meta-analysis of studies comparing TST vs IGRA for detecting latent TB across a variety of patient populations determined that TST sensitivity was poorly concordant across studies with a pooled estimate of $77 \%$. Pooled estimate of specificity was 97\% in non-Bacillus Calmette-Guérin (BCG)-vaccinated populations but only $77 \%$ in BCG-vaccinated populations. The IGRA had a pooled sensitivity of $76 \%$ and a specificity of $99 \%$ in non-BCG-vaccinated populations and $96 \%$ in BCG-vaccinated populations. ${ }^{26}$

Few studies have examined how the presence of psoriasis affects the IGRA. Garcovich et al used the IGRA as gold standard and compared its clinical correlation with TST in patients with psoriasis before biologic therapy, at 6 months and 12 months. Results showed agreement to be moderate ( $K=0.408)$ at screening, $\operatorname{good}(K=0.734)$ at 6 months, and fair (0.328) at 12 months. ${ }^{27}$ A 2009 retrospective study of 50 patients with psoriasis found the agreement between the IGRA and TST to be fair $(K=0.33) .{ }^{28}$

One explanation for poor agreement of TST with IGRA in patients with psoriasis is that a skin test performed on patient with an underlying inflammatory skin condition is more likely to give a false-positive result. ${ }^{25}$

Screening and monitoring for TB are essential, given the underlying mechanisms of action of the different biologic therapies. All clinicians must be aware of the risk of the reactivation of latent $\mathrm{TB}$, despite initial treatment with anti-TB therapy, always maintaining a high level of suspicion, particularly as the presentation is often atypical. ${ }^{5}$

Current recommendations for pretreatment screening are highlighted in Table 1. The main disparity between guidelines is regarding annual screening during the treatment. The BAD advises IGRA testing if a patient has been at high risk of TB exposure, AAD recommends yearly TST testing as standard with more frequent testing in high-risk groups, and EADV recommends both TST and IGRA testing on a yearly basis.

The EADV rationale for dual testing is that patients on biologics will have a degree of immunosuppression, therefore increasing the risk of false negatives in both the IGRA and TST as they both require a T-cell immune response. Dual testing is therefore a "belt-and-braces" approach to screening. ${ }^{29}$

Given that evidence demonstrates IGRA to be the superior test it would seem the most appropriate pretreatment and monitoring test for TB infection. This is supported by NICE guidelines for TB, which state that the IGRA as a standalone test is sufficient in those with immunosuppression. Furthermore, in countries with high BCG vaccination rates, false positives in TST are more likely and could lead to inappropriate, prolonged courses of anti-TB medications. ${ }^{30}$ Annual screening is the current consensus with regular patient reviews to assess possible exposures. The authors' opinion is for both pretreatment and annual IGRA testing. Patients with latent TB should be treated with chemoprophylaxis, eg, 6 months of isoniazid which is the NICE-recommended 
treatment. The consensus is the treatment should be for 1 month prior to the commencement of immunosuppression, but no firm data exist to support this contention. ${ }^{31}$

\section{Human immunodeficiency virus}

There are few case reports regarding human immunodeficiency virus (HIV) and treatment with TNF inhibitors. From the limited data available, treatment with TNF inhibitors did not increase viral load. ${ }^{32}$

\section{Hepatitis}

\section{Hepatitis B}

A total of 350 million patients are estimated to have chronic hepatitis B virus (HBV) infection worldwide. ${ }^{33}$ After inoculation of the virus, there can be four possible disease states: acute, chronic, occult, and resolved. The risk of reactivation of HBV is variable depending on whether a patient's hepatitis $\mathrm{B}$ is:

- chronic (HBsAg and $\mathrm{HBcAb}$ positive, $\mathrm{HBsAb}$ negative, and subclassified into active or inactive carriers depending on viral load),

- occult (HBsAg negative, HBcAb positive), or

- resolved (HBsAb positive). ${ }^{34,35}$

A 2011 analysis of hepatitis B reactivation in patients taking TNF inhibitors for various autoimmune diseases reported a reactivation rate of $39 \%$ in patients with chronic hepatitis $B$ and a reactivation rate of only $5 \%$ in patients with occult hepatitis B. The study also highlighted the reduced rates of reactivation in patients taking antiviral therapy. ${ }^{36}$

A small retrospective analysis looking at the reactivation of hepatitis B in 62 occult carriers undergoing treatment with either etanercept, infliximab, or adalimumab for psoriasis did not observe any signs of $\mathrm{HBV}$ reactivation. In one patient, there was a reappearance of HBsAg after 10 months of discontinuation from the previous course of treatment. Biological therapy was reintroduced in association with antiretroviral therapy with no adverse events occurring. ${ }^{37}$

These findings are supported by a 2015 retrospective analysis by Sanz-Bueno et al, ${ }^{22}$ which identified 20 patients who were treated for psoriasis with a biologic agent (TNF inhibitor or ustekinumab) and had evidence of prior infection with hepatitis B (defined as the presence of anti-HBc, the absence of HBsAg, and the presence or absence of anti-HBs) and assessed the cases for evidence of HBV reactivation. Hepatitis B reactivation was defined as the detection of $\mathrm{HBV}$ DNA in blood \pm conversion to $\mathrm{HBsAg}+$, and it was found that over a median 40-month follow-up there were no cases of reactivation of $\mathrm{HBV}$ despite none of the patients receiving chemoprophylaxis. This study combined its data with data from other studies of patients with psoriasis with past HBV infection and concluded that in patients with prior hepatitis $\mathrm{B}$ (but no evidence of chronic carriage) there is a maximum estimated risk of reactivation of 2.7 per 100 patient-years for a mean follow-up of 30 months and advised regular monitoring of alanine transaminase (ALT) and HBV viral load in this cohort of patients. Monitoring intervals were not commented on. ${ }^{38}$

\section{Hepatitis C}

The review of Pompili et al assessed the effect of TNF inhibitors over a median of 1.2 years in 216 patients with chronic hepatitis $\mathrm{C}$, suffering various autoimmune conditions including psoriasis, where three of whom had withdrawn the treatment on account of deteriorating hepatic function. ${ }^{39}$

Data specifically related to psoriasis are sparse. A 2013 retrospective study of 15 chronic hepatitis $\mathrm{C}$ sufferers treated for psoriatic arthritis with TNF inhibitors showed no worsening of viral load or liver markers over a 12-month follow-up period. $^{40}$

There is limited evidence regarding the safety of ustekinumab treatment in patients with psoriasis and concomitant $\mathrm{HBV}$ and hepatitis $\mathrm{C}$ virus infection. In a small retrospective, multicentre study of 25 patients with concurrent hepatitis $\mathrm{C}$ (20 patients) and hepatitis B infection (five patients), four patients underwent treatment with ustekinumab. In those with hepatitis B infection, all of which were being treated with antiviral therapy, no patients had significant changes in their viral load. Furthermore, the patients with hepatitis $\mathrm{C}$ infection receiving treatment with ustekinumab also had no significant changes in their viral load or liver function tests. ${ }^{41}$

There are no available data with which to draw conclusions about the safety of secukinumab for the treatment of patients with psoriasis who have hepatitis $\mathrm{B}$ or $\mathrm{C}$, nor the need for hepatitis screening.

\section{Guidelines for assessment and monitoring}

All guidelines support performing a hepatitis panel prior to the treatment. HIV screening was also supported by the BAD and EADV but not by the AAD. The evidence is limited to small study populations, but particularly for hepatitis B there is evidence to support prescreening. The risk of reactivation in patients with hepatitis B depends on a number of factors including whether patients are active/inactive/occult carriers, the number of comorbidities, and use of immunosuppression. However, there is no doubt that the risk exists and should be considered before starting the treatment with TNF inhibitor 
therapy. Consultation with a hepatologist would be advised prior to initiating the treatment.

Given that data on ustekinumab are minimal, it would be advisable to follow the same screening guidance as for the TNF inhibitors.

New EADV guidelines state infliximab and adalimumab are absolutely contraindicated in patients with chronic hepatitis B but that etanercept is only relatively contraindicated. The review of cited literature in the EADV guidelines as well as independent literature search has not uncovered any research supporting differing levels of risk between the TNF inhibitors. $^{21}$

Literature suggests that biologic therapy is relatively safe in patients with prior HBV infection (who therefore could be occult carriers) but advises monitoring of ALT and HBV viral load to identify seroconversion. Optimum screening intervals have yet to be determined.

\section{Cardiovascular disease}

Ryan et al published a meta-analysis in 2011 focusing on the incidence of cardiovascular disease in patients treated with TNF inhibitors or ustekinumab for psoriasis. ${ }^{36}$ A total of 22 randomized controlled trials (RCTs) were identified with a combined patient cohort of 10,183 patients. The primary outcome measure of the meta-analysis was the incidence of Major Adverse Cardiovascular Events (MACE), defined as one of the following: myocardial infarction, cerebrovascular accident, or cardiovascular death. The meta-analysis did not find any association between the use of TNF inhibitors or ustekinumab and increased risk of MACE. ${ }^{42}$ Further research by Gottlieb et al and Hugh et al supported these findings, reporting no increased risk of MACE with biologics when compared against patients with psoriasis receiving nonbiologic therapies. ${ }^{43,44}$

Tzellos et al conducted a meta-analysis looking at the anti-IL12-23s (ustekinumab and briakinumab) and the number of MACEs. Nine RCTs were identified: five using ustekinumab as the intervention and four using briakinumab. No individual RCT showed a statistically significant increased number of MACEs in the treatment arm; however, when all RCTs were analyzed together anti-IL12-23s were found to have a statistically significant increased risk (odds ratio [OR]: 4.23; 95\% CI: $1.07-16.75 ; P=0.04) .{ }^{45}$

Both the secukinumab trials, ERASURE and FIXTURE, recorded the incidence of MACE over a 52-week follow-up period. The reported incidence of MACE per 100 patientyears for secukinumab $300 \mathrm{mg}$, secukinumab $150 \mathrm{mg}$, and placebo were $0.7,0.7$, and 0 , respectively, in the ERASURE study and 0,0.4, and 0, respectively, in the FIXTURE study. The statistical significance of these figures was not commented on. ${ }^{14}$

\section{Guidelines for assessment and monitoring}

All guidelines recommend that TNF inhibitors are contraindicated in patients with New York Heart Association (NYHA) class III or IV heart failure and advise caution in those with NYHA classification stage I or II disease. The British and American guidelines recommend echocardiography for patients with NYHA I or II heart failure and advise physicians to consider alternatives to biologic therapy if a patient has left ventricular ejection fraction (LVEF) $<50 \%$. This guidance is borne out of findings from the 1999 Anti-TNF Therapy Against Congestive Heart Failure (ATTACH) trial that was conducted to determine whether infliximab would improve outcomes in 150 patients with heart failure with an NYHA classification of III or VI and a left ventricular ejection fraction of $<35 \%$. The trial showed increased rates of all-cause mortality or hospitalization due to heart failure compared with placebo in the infliximab $10 \mathrm{mg} / \mathrm{kg}$ arm of the trial. ${ }^{46}$

Subsequent studies have failed to show association between TNF inhibitors and worsening of heart failure across a variety of patient groups and data in psoriasis-specific patient groups have shown no such association. ${ }^{7,43,47}$ There are no trials associating ustekinumab or secukinumab with heart failure. In those with NYHA I or II heart failure, it is likely that benefits of treatment would outweigh risk.

There is no current evidence to support an increased risk of MACE with any of the biologic therapies currently licensed for psoriasis; however, the results of the Tzellos study suggest that further investigation into the risk of MACE with IL12-23 inhibitors would be valuable.

\section{Neurological disease}

As of present, pharmacovigilance data have shown no association between TNF inhibitors and demyelinating disease. Current guidance from the BAD recommends avoiding their use in patients with a history of demyelinating disease or in those with a first-degree relative with a history of demyelinating disease, based on numerous case reports of exacerbations of multiple sclerosis (MS) or ne-onset central demyelination. ${ }^{5,48}$

Initial concerns over the TNF inhibitors arose from a study in 1999 assessing lenercept in MS, which resulted in early deterioration of the MS. ${ }^{49}$ 
A 2009 Phase II, multicentre, double-blind, placebocontrolled RCT looked at the use of ustekinumab with relapsing remitting MS. This trial demonstrated no evidence of worsening neurological disease. ${ }^{50}$

\section{Guidelines for assessment and monitoring}

The current, conflicting evidence suggests that TNF inhibitors can induce/exacerbate the MS. Given the uncertainty surrounding this link, the suggestion from all three guidelines to avoid TNF inhibitors in patients with MS seems prudent. This position is reinforced by the literature and given that there are alternative biologics (ustekinumab); the risk would be unacceptable. The most recent European guidelines now recommend the use of ustekinumab in concomitant demyelinating conditions. ${ }^{21}$

\section{Malignancy}

The evidence base on which current recommendations for screening in psoriasis patients commencing treatment with biologics in malignancy is limited. Increased rates of tobacco and alcohol use in patients with psoriasis mean that as a group they have higher rates of certain cancers (respiratory tract, digestive tract, urinary tract cancers). ${ }^{51}$ The same metaanalysis showed a higher risk of squamous cell carcinoma in patients with psoriasis (standardized incidence ratio of 5.3), possibly linked to their exposure to 8-methoxypsoralenultraviolet A, ciclosporin, and methotrexate. Patients with psoriasis were also found to be at higher risk of nonmelanoma skin cancer. ${ }^{51}$

Dommasch et al in a meta-analysis of randomized, placebo-controlled trials, which looked at the risk of malignancy in 6,810 adults with psoriatic disease treated with TNF inhibitors, found no statistically increased risk of cancer, except for nonmelanoma skin cancer. ${ }^{52}$

Most of the researches into association between TNF inhibitors and cancer are from studies of patients who are treated for rheumatoid arthritis. Although safety may be inferred from such data, it is not ideal to assume that it is applicable to other disease states.

In 2006, Bongartz et al published a meta-analysis of nine RCTs that included data from 3,493 patients with rheumatoid arthritis treated with either infliximab or adalimumab vs placebo and concluded that TNF inhibitors were associated with an increased risk of all cancers (with a large number of the cancers found to be nonmelanoma skin cancer). ${ }^{53}$

However, subsequently published data from the Swedish and German rheumatology biologic registers, ${ }^{54,55}$ data collated from 2001 to 2011 by the British Society of Rheumatology and Biologics Registry, and Le Blay et al reported no evidence to suggest that TNF inhibitors confer an increased risk of cancer compared with biologic-naïve patients, except a possible increased risk of nonmelanoma skin cancerin the study by Le Blay et al. ${ }^{56,57}$ Similarly, the British Rheumatology register showed an increased rate of recurrence of melanoma, but no other cancers in patients taking TNF inhibitors.

There have previously been concerns regarding lymphoma in patients taking TNF inhibitors, but the 2011 Cochrane review into adverse effects of biologics for a number of autoimmune conditions including psoriasis found no evidence associating TNF inhibitors with increased incidence of lymphoma. However, the authors report that given the low numbers of lymphoma cases identified, these results should be interpreted with caution. ${ }^{7}$

Increased rates of high-grade cervical intraepithelial neoplasia (CIN) and cervical cancer have been seen in females who are immunosuppressed due to HIV infection or because of immunosuppressive therapy post renal transplant. ${ }^{58}$ The concern that immunosuppression increases the risk of cervical cancer prompted Kim et al to conduct a cohort study comparing 133,333 females with a variety of inflammatory diseases including psoriasis with 533,332 controls. ${ }^{59}$ Results showed that among 34,665 patients with psoriasis there was a crude incidence rate of high-grade CIN or cervical cancer of 82.2 per 100,000 patient-years compared with rates in the control group of 73.4 per 100,000 patient-years with a hazard ratio of 0.96 (95\% CI: $0.73-1.27)$, therefore showing no statistical significance. ${ }^{59}$

A 2015 meta-analysis assessing the risk of high-grade $\mathrm{CIN}$ or cervical cancer in patients with inflammatory bowel disease receiving immunosuppressive therapy found that immunosuppressed IBD patients had significantly higher rates of high-grade CIN/cervical cancer than females in the general population. This prompted authors to advise increased screening frequency. ${ }^{60}$

There is a scarcity of literature with regard to CIN/cervical cancer risk in patients with psoriasis and patients taking biologic therapies. Until further evidence is available, patients should be advised to strictly adhere to national screening programs for cervical cancer and to consider human papilloma virus vaccination prior to starting a biologic therapy. In patients with CIN or previous cervical cancer, a gynecological opinion should be sought prior to the decision to start a biologic therapy.

\section{Guidelines for assessment and monitoring}

Current guidelines are in agreement that if a patient has had cancer in the last 5 years then TNF-inhibitors and ustekinumab are relatively contraindicated. 
Evidence suggests that patients are not at increased risk of any de novo cancer with TNF inhibitors, with the possible exceptions of nonmelanoma skin cancer and melanoma skin cancer. There is evidence that melanoma has increased recurrence rate associated with TNF inhibitors.

BAD guidelines suggest "monitoring," every 3 months for cancer, although they do not specify what "monitoring" should entail. Evidence would certainly support monitoring for melanoma and nonmelanoma skin cancers in patients receiving biologics, especially in patients with previous such cancers or those considered to be of high risk.

\section{Laboratory testing}

$\mathrm{BAD}, \mathrm{EADV}$, and $\mathrm{AAD}$ guidelines differ significantly in respect of test selection before treatment and retesting frequency during the treatment. van Lümig et al reviewed 162 patients over 5 years receiving either etanercept or adalimumab. ${ }^{61}$ Of the 62 patients, 38 (24\%) of the adalimumab group and eight (14\%) of the etanercept group developed one or more grade 3 (severe/medically significant, but not life-threatening) or grade 4 (life-threatening) laboratory abnormality. Nine percent of patients developed hematological abnormalities, none of which led to permanent discontinuation of treatment. ${ }^{60}$ No evidence of renal impairment was found, it has however been demonstrated in the clinical trials with adalimumab. ${ }^{62}$

The summary of product characteristics for both etanercept and adalimumab reports elevated liver enzymes during the treatment. ${ }^{62,63}$ In the aforementioned cohort, a significant rise in the mean ALT was demonstrated; however, these values did not exceed the upper limit of the normal reference range. ${ }^{61}$ The Italian PSOCARE registry found that that in patients treated with infliximab, it was associated with the risk of doubling the upper limit of aspartate aminotransferase (OR: 1.87) and ALT (OR: 1.74). No change in serum aspartate aminotransferase or ALT levels was seen in those treated with adalimumab or etanercept at baseline, weeks 8 or 16 during the treatment. ${ }^{64}$

The BAD guidelines currently support the use of antinuclear antibody testing, which was observed in $<4 \%$ of patients in the cohort study, with no patients developing clinical symptoms. ${ }^{61}$ Previous literature has shown, however, that seroconversion of anti-nuclear antibodies (ANA) during TNF inhibitor therapy can result in a lupus-like syndrome, the level of which can be reassessed if clinical symptoms develop. ${ }^{65}$ However, the evidence is poor and larger prospective studies are required.
No studies were found assessing laboratory testing and monitoring for patients with psoriasis treated with secukinumab or ustekinumab.

\section{Guidelines for assessment and monitoring}

The BAD, EADV, and AAD recommend undertaking a number of screening laboratory investigations at baseline and regular intervals throughout the treatment, which is supported by little or no evidence. There needs to be further larger prospective studies to provide further evidence about which laboratory testing is appropriate and at what intervals.

\section{Pregnancy}

Bogas and Leandro reviewed cases of biologic use in pregnancy for a variety of autoimmune conditions including plaque psoriasis. The review concluded that the lack of data was a significant limitation to drawing conclusions about the safety of TNF inhibitors in pregnancy, but that the risk appeared to be low, with no significant increase in congenital malformations or complications seen in comparison with rates in the general population. ${ }^{6}{ }^{6}$ These findings are supported by a Spanish retrospective cohort study in IBD patients and in the study by Chambers and Johnson, which found no association between TNF inhibitors and unfavorable pregnancy outcomes. ${ }^{67,68}$

In contrast, data from the British Society of Rheumatology and Biologics Registry have suggested an increased rate of early spontaneous miscarriage in patients taking TNF inhibitors, but results are confounded by concomitant methotrexate use and severity of rheumatological disease. ${ }^{69}$

A total of 31 cases of pregnancy associated with maternal ustekinumab use have been found in pooled clinical trial data over a period of 4 years, with no incidence of fetal malformation or death reported. ${ }^{70}$

As of yet, there is insufficient evidence of the safety profile for ustekinumab and secukinumab with which to draw conclusions about their use in pregnancy.

\section{Guidelines for assessment and monitoring}

BAD guidelines advise discontinuing TNF inhibitors in pregnancy, whereas guidance from the AAD does not offer a recommendation. European guidelines state that treatment with adalimumab and infliximab is absolutely contraindicated, whereas etanercept and ustekinumab are relatively contraindicated.

There is currently no conclusive evidence linking TNF inhibitors to maternal complications in pregnancy or congenital malformations, ${ }^{70}$ and EADV guidance recommends 
that, for those with severe psoriasis who require a systemic agent, etanercept should be regarded as first line where the benefit outweighs the risk.

Currently, in the UK, ciclosporin is the first-line systemic treatment over biologic therapy; however, given the accumulating evidence over the safety of TNF inhibitors in pregnancy, they may be recommended to be continued in the first and second trimesters on a case-by-case basis in future iterations of biologic therapy guidelines for psoriasis. Further results from observational registries in patients with psoriasis on biologic therapies will help clarify the risk of maternal and newborn complications.

\section{Vaccinations}

Given that biologic agents target the immune system, using all available approaches to prevent infection is vital. Current guidance from the AAD, EDAV, and BAD all recommend vaccination prior to the commencement of a systemic therapy if possible with pneumococcal, influenza, tetanus-diphtheria, and hepatitis A/B depending upon the local guidance. Hepatitis B vaccination is supported by the AAD and EADV only. Live vaccines including BCG vaccine, varicella-zoster virus, yellow fever, and measlesmumps-rubella vaccinations are contraindicated in patients treated with biologic agents. Currently, neither the EADV,

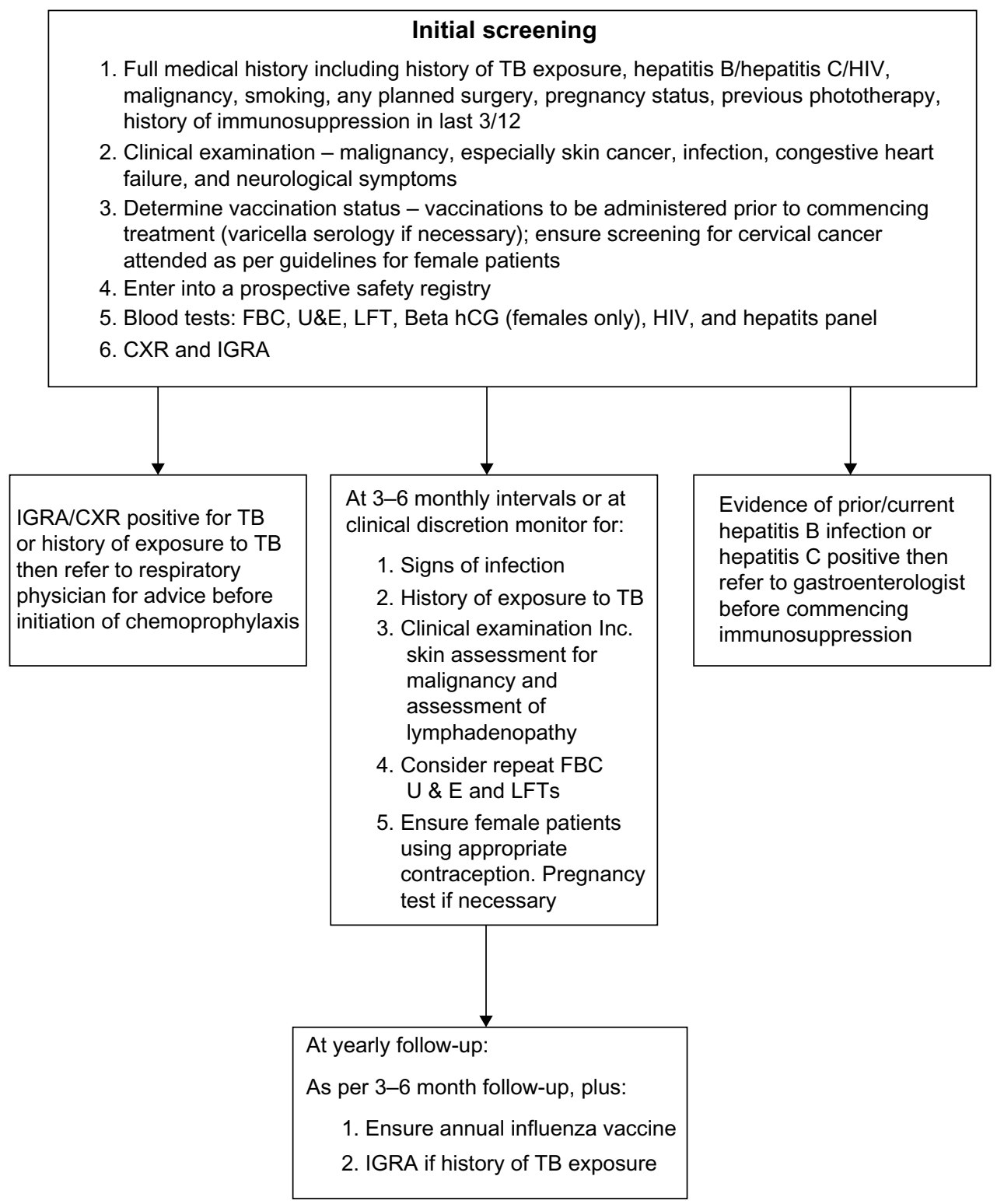

Figure I Suggested algorithm for screening and monitoring of adverse effects associated with biologics.

Abbreviations: HIV, human immunodeficiency virus; IGRA, interferon gamma release assay; TB, tuberculosis; CXR, chest X-ray; U\&E, urea and electrolytes; HcG, human chorionic gonadotropin. 
$\mathrm{AAD}$, or BAD guidelines recommend prior vaccination with herpes zoster; however, a recent review that evaluated the risk of herpes zoster during the treatment with etanercept, adalimumab, infliximab, and ustekinumab in psoriasis and other inflammatory conditions found infliximab to be associated with an increased risk of herpes zoster, but the risk with the other biologics remains controversial, therefore vaccination should be considered. ${ }^{71}$

At present, no data have been published specific to patients with psoriasis. Data from other population groups, however, have demonstrated that the use of TNF- $\alpha$ inhibitors does not influence the effectiveness of vaccination against influenza $A$ but may impair the immune response to influenza $\mathrm{B}$ and the pneumococcal vaccine. The immune response, however, in those receiving ustekinumab is not believed to be impaired. ${ }^{72,73}$

Given the current evidence and the increased theoretical risk of infection in those receiving treatment with a biologic, the consensus is the routine vaccination in line with local guidance with inactivated vaccines prior to the treatment and annual influenza during the treatment if required.

\section{Conclusion}

From this review of the current literature and guidelines, it is evident that there are areas of routine screening and monitoring, which are recommended in current practice, that are supported by limited evidence. It is, no doubt, difficult for professional bodies to make recommendations on the frequency screening and monitoring of low frequency of adverse events, when the data to underpin these recommendations are limited. Furthermore, clinicians need to consider the associated health care costs of routine testing and the risk of detecting false positives, leading to further investigation and morbidity. This highlights the need for evidence from real-world observational cohorts/registries to evaluate the true risk of various adverse events in those with psoriasis undergoing treatment with biologics.

Despite the various adverse events presented here, it is important to note that biologic therapies have a better and more well-defined safety profile than many alternative conventional systemic therapies for psoriasis and other autoimmune diseases.

This paper draws together evidence from major psoriasis biologic therapy guidelines as an accessible overall summary of how these drugs should be monitored (Figure 1). Given the gaps in knowledge which are currently undergoing investigation in various biologic registries around the world, it is likely that recommendations for assessment and monitoring of adverse events will also be updated based on better evidence accordingly in the near future.

\section{Disclosure}

The authors report no conflicts of interest in this work.

\section{References}

1. Menter A, Gottlieb A, Feldman SR, et al. Guidelines of care for the management of psoriasis and psoriatic arthritis: section 1. Overview of psoriasis and guidelines of care for the treatment of psoriasis with biologics. J Am Acad Dermatol. 2008;58(5):826-850.

2. Rapp SR, Feldman SR, Exum ML, Fleischer AB Jr, Reboussin DM. Psoriasis causes as much disability as other major medical diseases. J Am Acad Dermatol. 1999;41(3 pt 1):401-407.

3. NICE.org.uk [webpage on the Internet]. National Institute of Clinical Excellence. Psoriasis Guidelines [updated August 2013; cited September 1, 2015]. Available from: https://www.nice.org.uk/guidance/ qs40. Accessed January 21, 2016.

4. Zachariae H. Prevalence of joint disease in patients with psoriasis: implications for therapy. Am J Clin Dermatol. 2003;4(7):441-447.

5. Smith $\mathrm{CH}$, Anstey AV, Barker JN, et al; Chair of Guideline Group. British Association of Dermatologists' guidelines for biologic interventions for psoriasis 2009. Br J Dermatol. 2009;161(5):987-1019.

6. Garcia-Doval I, Carretero G, Vanaclocha F. Risk of serious adverse events associated with biologic and nonbiologic psoriasis systemic therapy: patients ineligible vs eligible for randomized controlled trials. Arch Dermatol. 2012;148(4):463-470.

7. Singh JA, Wells GA, Christensen R, et al. Adverse effects of biologics: a network meta-analysis and Cochrane overview. Cochrane Database Syst Rev. 2011;(2):CD008794.

8. Kothary N, Diak IL, Brinker A, Bezabeh S, Avigan M, Dal Pan G. Progressive multifocal leukoencephalopathy associated with efalizumab use in psoriasis patients. J Am Acad Dermatol. 2011;65(3):546-551.

9. Burden AD, Warren RB, Kleyn CE, et al. The British Association of Dermatologists' Biologic Interventions Register (BADBIR): design, methodology and objectives. Br J Dermatol. 2012;166(3):545-554.

10. Iskandar IY, Ashcroft DM, Warren RB. Demographics and disease characteristics of patients with psoriasis enrolled in the British Association of Dermatologists Biologic Interventions Register. Br J Dermatol. 2015;173(2):510-518.

11. Ali T, Kaitha S, Mahmood S, Ftesi A, Stone J, Bronze MS. Clinical use of anti-TNF therapy and increased risk of infections. Drug Healthc Patient Saf. 2013;5:79-99.

12. Kalb RE, Fiorentino DF, Lebwohl MG. Risk of serious infection with biologic and systemic treatment of psoriasis: results from the psoriasis longitudinal assessment and registry (PSOLAR). JAMA Dermatol. 2015;151(9):961-969.

13. Reich K, Papp KA, Griffiths CE. An update on the long-term safety experience of ustekinumab: results from the psoriasis clinical development program with up to four years of follow-up. J Drugs Dermatol. 2012;11(3):300-312.

14. Langley RG, Elewski BE, Lebwohl M, et al; ERASURE Study Group; FIXTURE Study Group. Secukinumab in plaque psoriasis - results of two phase 3 trials. N Engl J Med. 2014;371(4):326-338.

15. Sánchez-Moya AI, García-Doval I, Carretero G, BIOBADADERM Study Group. Latent tuberculosis infection and active tuberculosis in patients with psoriasis: a study on the incidence of tuberculosis and the prevalence of latent tuberculosis disease in patients with moderatesevere psoriasis in Spain. BIOBADADERM registry. J Eur Acad Dermatol Venereol. 2013;27(11):1366-1374.

16. Sánchez-Moya AI, Dauden E. Incidence of tuberculosis infection in psoriatic patients on anti-TNF therapy: report of a case series with 144 patients. J Eur Acad Dermatol Venereol. 2011;25(6):730-733. 
17. Ergun T, Seckin D, Baskan Bulbul E, et al. The risk of tuberculosis in patients with psoriasis treated with anti-tumor necrosis factor agents. Int J Dermatol. 2015;54(5):594-599.

18. NICE.org.uk [webpage on the Internet]. National Institute of Clinical Excellence. Ustekinumab for the Treatment of Adults with Moderate to Severe Psoriasis [updated September 2009; cited September 1, 2015]. Available from: https://www.nice.org.uk/guidance/ta180. Accessed January 21, 2016.

19. Tsai TF, Ho V, Song M, et al; PHOENIX 1, PHOENIX 2, ACCEPT, PEARL and Japanese Ustekinumab Study Groups. The safety of ustekinumab treatment in patients with moderate-to-severe psoriasis and latent tuberculosis infection. Br J Dermatol. 2012;167(5): $1145-1152$.

20. Tsai T-F, Blauvelt A, Fox T, Gong Y, Huang J. Secukinumab treatment shows no evidence for reactivation of previous or latent TB infection in subjects with psoriasis: a pooled phase 3 safety analysis. J Am Acad Dermatol. 2015;72(5):AB251.

21. Nast A, Gisondi P, Ormerod AD, et al. European S3-Guidelines on the systemic treatment of psoriasis vulgaris - update 2015 - short version EDF in cooperation with EADV and IPC. J Eur Acad Dermatol Venereol. 2015;29(12):2277-2294.

22. Sanz-Bueno J, Vanalclocha F, Garcia-Doval I et al. Risk of reactivation of Hepatitis B virus infection in Psoriasis Patients Treated with Biologics: A retrospective analysis of 20 cases from the BIOBADADERM database. Actas Dermo-sifiliograficas. 2015;106(6):477-472.

23. Mohan AK, Coté TR, Block JA, Manadan AM, Siegel JN, Braun MM. Tuberculosis following the use of etanercept, a tumor necrosis factor inhibitor. Clin Infect Dis. 2004;39(3):295-299.

24. NICE.org.uk [webpage on the Internet]. National Institute of Clinical Excellence. Secukinumab for Treating Moderate to Severe Plaque Psoriasis [updated July 2015; cited September 1, 2015]. Available from https://www.nice.org.uk/guidance/ta350. Accessed January 21, 2016.

25. Sivamani RK, Goodarzi H, Garcia MS, et al. Biologic therapies in the treatment of psoriasis: a comprehensive evidence-based basic science and clinical review and a practical guide to tuberculosis monitoring. Clin Rev Allergy Immunol. 2013;44(2):121-140.

26. Pai M, Zwerling A, Menzies D. Systematic review: T-cell-based assays for the diagnosis of latent tuberculosis infection: an update. Ann Intern Med. 2008;149(3):177-184.

27. Garcovich S, Ruggeri A, D’Agostino M, et al. Clinical applicability of Quantiferon-TB-Gold testing in psoriasis patients during long-term anti-TNF-alpha treatment: a prospective, observational study. $J$ Eur Acad Dermatol Venereol. 2012;26(12):1572-1576.

28. Laffitte E, Janssens JP, Roux-Lombard P, et al. Tuberculosis screening in patients with psoriasis before antitumour necrosis factor therapy: comparison of an interferon-gamma release assay vs tuberculin skin test. Br J Dermatol. 2009;161(4):797-800.

29. Tagmouti S, Slater M, Benedetti A, et al. Reproducibility of interferon gamma (IFN- $\gamma$ ) release assays. A systematic review. Ann Am Thorac Soc. 2014;11(8):1267-1276.

30. NICE.org.uk [webpage on the Internet]. National Institute of Clinical Excellence: Clinical Diagnosis and Management of Tuberculosis, and Measures for Its Prevention and Control [updated March 2011 cited September 1, 2015]. Available from: https://www.nice.org.uk/ guidance/cg117. Accessed January 21, 2016.

31. Solovic I, Sester M, Gomez-Reino JJ, et al. The risk of tuberculosis related to tumour necrosis factor antagonist therapies: a TBNET consensus statement. Eur Respir J. 2010;36(5):1185-1206.

32. Domm S, Cinatl J, Mrowietz U. The impact of treatment with tumour necrosis factor-alpha antagonists on the course of chronic viral infections: a review of the literature. Br J Dermatol. 2008;159(6): 1217-1228.

33. WHO.int [webpage on the Internet]. World Health Organisation. Hepatitis B. [updated July 2015; cited September 1, 2015]. Available from: http://www.who.int/mediacentre/factsheets/fs204/en/. Accessed January 21, 2016.
34. Ahn CS, Dothard EH, Garner ML, Feldman SR, Huang WW. To test or not to test? An updated evidence-based assessment of the value of screening and monitoring tests when using systemic biologic agents to treat psoriasis and psoriatic arthritis. J Am Acad Dermatol. 2015;73(3):420.e-428.e.

35. Motaparthi K, Stanisic V, Van Voorhees AS, Lebwohl MG, Hsu S; Medical Board of the National Psoriasis. From the Medical Board of the National Psoriasis Foundation: recommendations for screening for hepatitis B infection prior to initiating antitumor necrosis factor-alfa inhibitors or other immunosuppressive agents in patients with psoriasis. J Am Acad Dermatol. 2014;70(1): 178-186.

36. Pérez-Alvarez R, Díaz-Lagares C, García-Hernández F, et al; BIOGEAS Study Group. Hepatitis B virus (HBV) reactivation in patients receiving tumor necrosis factor (TNF)-targeted therapy: analysis of 257 cases. Medicine (Baltimore). 2011;90(6):359-371.

37. Cassano N, Mastrandrea V, Principi M, et al. Anti-tumor necrosis factor treatment in occult hepatitis B virus infection: a retrospective analysis of 62 patients with psoriatic disease. J Biol Regul Homeost Agents. 2011;25(2):285-289.

38. Keane J, Gershon S, Wise RP, et al. Tuberculosis associated with infliximab, a tumor necrosis factor alpha-neutralizing agent. $N$ Engl J Med. 2001;345(15):1098-1104

39. Pompili M, Biolato M, Miele L, Grieco A. Tumor necrosis factor- $\alpha$ inhibitors and chronic hepatitis $\mathrm{C}$ : a comprehensive literature review. World J Gastroenterol. 2013;19(44):7867-7873.

40. Costa L, Caso F, Atteno M, et al. Long-term safety of anti-TNF- $\alpha$ in PsA patients with concomitant HCV infection: a retrospective observational multicenter study on 15 patients. Clin Rheumatol. 2014;33(2):273-276.

41. Navarro R, Vilarrasa E, Herranz P, et al. Safety and effectiveness of ustekinumab and antitumour necrosis factor therapy in patients with psoriasis and chronic viral hepatitis $\mathrm{B}$ or $\mathrm{C}$ : a retrospective, multicentre study in a clinical setting. Br J Dermatol. 2013;168(3): 609-616.

42. Ryan C, Leonardi CL, Krueger JG, et al. Association between biologic therapies for chronic plaque psoriasis and cardiovascular events: a meta-analysis of randomized controlled trials. JAMA. 2011;306(8):864-871.

43. Gottlieb AB, Kalb RE, Langley RG, et al. Safety observations in 12095 patients with psoriasis enrolled in an international registry (PSOLAR): experience with infliximab and other systemic and biologic therapies. J Drugs Dermatol. 2014;13(12):1441-1448.

44. Hugh J, Van Voorhees AS, Nijhawan RI, et al. From the Medical Board of the National Psoriasis Foundation: the risk of cardiovascular disease in individuals with psoriasis and the potential impact of current therapies. J Am Acad Dermatol. 2014;70(1):168-177.

45. Tzellos T, Kyrgidis A, Zouboulis CC. Re-evaluation of the risk for major adverse cardiovascular events in patients treated with antiIL-12/23 biological agents for chronic plaque psoriasis: a meta-analysis of randomized controlled trials. J Eur Acad Dermatol Venereol. 2013;27(5):622-627.

46. Chung ES, Packer M, Lo KH, Fasanmade AA, Willerson JT; AntiTNF Therapy Against Congestive Heart Failure Investigators. Randomized, double-blind, placebo-controlled, pilot trial of infliximab, a chimeric monoclonal antibody to tumor necrosis factor-alpha, in patients with moderate-to-severe heart failure: results of the anti-TNF Therapy Against Congestive Heart Failure (ATTACH) trial. Circulation . 2003;107(25):3133-3140.

47. Mann DL, McMurray JJ, Packer M, et al. Targeted anticytokine therapy in patients with chronic heart failure: results of the randomized etanercept worldwide evaluation (RENEWAL). Circulation. 2004;109(13):1594-1602.

48. Mahil SK, Andrews TC, Brierley C, Barker JN, Smith CH. Demyelination during tumour necrosis factor antagonist therapy for psoriasis: a case report and review of the literature. J Dermatolog Treat. $2013 ; 24(1): 38-49$ 
49. TNF neutralization in MS: results of a randomized, placebo-controlled multicenter study. The Lenercept Multiple Sclerosis Study Group and The University of British Columbia MS/MRI Analysis Group. Neurology. 1999;53(3):457-465.

50. Segal BM, Constantinescu CS, Raychaudhuri A, et al; Ustekinumab MS Investigators. Repeated subcutaneous injections of IL12/23 p40 neutralising antibody, ustekinumab, in patients with relapsing-remitting multiple sclerosis: a phase II, double-blind, placebo-controlled, randomised, dose-ranging study. Lancet Neurol. 2008;7(9):796-804.

51. Pouplard C, Brenaut E, Horreau C, et al. Risk of cancer in psoriasis: a systematic review and meta-analysis of epidemiological studies. J Eur Acad Dermatol Venereol. 2013;27(suppl 3):36-46.

52. Dommasch ED, Abuabara K, Shin DB, Nguyen J, Troxel AB, Gelfand JM. The risk of infection and malignancy with tumor necrosis factor antagonists in adults with psoriatic disease: a systematic review and meta-analysis of randomized controlled trials. J Am Acad Dermatol. 2011;64(6):1035-1050.

53. Bongartz T, Sutton AJ, Sweeting MJ, Buchan I, Matteson EL, Montori V. Anti-TNF antibody therapy in rheumatoid arthritis and the risk of serious infections and malignancies: systematic review and metaanalysis of rare harmful effects in randomized controlled trials. JAMA. 2006;295(19):2275-2285.

54. Raaschou P, Simard JF, Neovius M, Askling J; Anti-Rheumatic Therapy in Sweden Study Group. Does cancer that occurs during or after antitumor necrosis factor therapy have a worse prognosis? A national assessment of overall and site-specific cancer survival in rheumatoid arthritis patients treated with biologic agents. Arthritis Rheum. 2011;63(7):1812-1822.

55. Strangfeld A, Hierse F, Rau R, et al. Risk of incident or recurrent malignancies among patients with rheumatoid arthritis exposed to biologic therapy in the German biologics register RABBIT. Arthritis Res Ther. 2010;12(1):R5.

56. Mercer LK, Lunt M, Low AL, et al. Risk of solid cancer in patients exposed to anti-tumour necrosis factor therapy: results from the British Society for Rheumatology Biologics Register for rheumatoid arthritis. Ann Rheum Dis. 2015;74(6):1087-1093.

57. Le Blay P, Mouterde G, Barnetche T, Morel J, Combe B. Risk of malignancy including non-melanoma skin cancers with anti-tumor necrosis factor therapy in patients with rheumatoid arthritis: meta-analysis of registries and systematic review of long-term extension studies. Clin Exp Rheumatol. 2012;30(5):756-764.

58. Dugue PA, Rebolj M, Garred P, Lynge E. Immunosuppression and risk of cervical cancer. Expert Rev Anticancer Ther. 2013;13(1):29-42.

59. Kim SC, Glynn RJ, Giovannucci E, et al. Risk of high-grade cervical dysplasia and cervical cancer in women with systemic inflammatory diseases: a population-based cohort study. Ann Rheum Dis. 2015;74(7):1360-1367.

60. Allegretti JR, Barnes EL, Cameron A. Are patients with inflammatory bowel disease on chronic immunosuppressive therapy at increased risk of cervical high-grade dysplasia/cancer? A meta-analysis. Inflamm Bowel Dis. 2015;21(5):1089-1097.
61. van Lümig PP, Driessen RJ, Roelofs-Thijssen MA, Boezeman JB, van de Kerkhof PC, de Jong EM. Relevance of laboratory investigations in monitoring patients with psoriasis on etanercept or adalimumab. Br J Dermatol. 2011;165(2):375-382.

62. Pfizer [webpage on the Internet]. Summary of Product Characteristics: Etanercept [cited September 1, 2015]. Available from: https://www. medicines.org.uk/emc/medicine/19162. Accessed January 21, 2016.

63. AbbVie [webpage on the Internet]. Summary of Product Characteristics: Adalimumab [cited September 1, 2015]. Available from: https://www. medicines.org.uk/emc/medicine/21201. Accessed January 21, 2016.

64. Gisondi P, Cazzaniga S, Chimenti S, et al; Psocare Study Group. Metabolic abnormalities associated with initiation of systemic treatment for psoriasis: evidence from the Italian Psocare Registry. J Eur Acad Dermatol Venereol. 2013;27(1):e30-e41.

65. Pink AE, Fonia A, Allen MH, Smith CH, Barker JN. Antinuclear antibodies associate with loss of response to antitumour necrosis factor-alpha therapy in psoriasis: a retrospective, observational study. Br J Dermatol. 2010;162:780-785.

66. Bogas M, Leandro MJ. Biologic therapy and pregnancy. A systematic literature review. Acta Reumatol Port. 2011;36(3):219-232.

67. Casanova MJ, Chaparro M, Domènech E, et al. Safety of thiopurines and anti-TNF- $\alpha$ drugs during pregnancy in patients with inflammatory bowel disease. Am J Gastroenterol. 2013;108(3):433-440.

68. Chambers CD, Johnson DL. Emerging data on the use of anti-tumor necrosis factor-alpha medications in pregnancy. Birth Defects Res $A$ Clin Mol Teratol. 2012;94(8):607-611.

69. Verstappen SM, King Y, Watson KD, Symmons DP, Hyrich KL; BSRBR Control Centre Consortium, BSR Biologics Register. BSRBR Control Centre Consortium BSRBR. Anti-TNF therapies and pregnancy: outcome of 130 pregnancies in the British Society for Rheumatology Biologics Register. Ann Rheum Dis. 2011;70(5):823-826.

70. Yiu ZZ, Griffiths CE, Warren RB. Safety of biological therapies for psoriasis: effects on reproductive potential and outcomes in male and female patients. Br J Dermatol. 2014;171(3):485-491.

71. Adelzadeh L, Jourabchi N, Wu JJ. The risk of herpes zoster during biological therapy for psoriasis and other inflammatory conditions. J Eur Acad Dermatol Venereol. 2014;28(7):846-852.

72. Salemi S, Picchianti-Diamanti A, Germano V, et al. Influenza vaccine administration in rheumatoid arthritis patients under treatment with TNFalpha blockers: safety and immunogenicity. Clin Immunol. 2010;134(2):113-120.

73. Brodmerkel $\mathrm{C}$, Wadman E, Langley RG, et al. Immune response to pneumococcus and tetanus toxoid in patients with moderate-to-severe psoriasis following long-term ustekinumab use. J Drugs Dermatol. 2013;12(10):1122-1129.
Psoriasis: Targets and Therapy

\section{Publish your work in this journal}

Psoriasis: Targets and Therapy is international, peer-reviewed, open access journal focusing on psoriasis, nail psoriasis, psoriatic arthritis and related conditions, identification of therapeutic targets and the optimal use of integrated treatment interventions to achieve improved outcomes and quality of life. The manuscript management system

\section{Dovepress}

is completely online and includes a very quick and fair peer-review system. Visit http://www.dovepress.com/testimonials.php to read real quotes from published authors. 